This item was submitted to Loughborough's Research Repository by the author.

Items in Figshare are protected by copyright, with all rights reserved, unless otherwise indicated.

\title{
Microscopic theory of solvent-mediated long-range forces: influence of wetting
}

PLEASE CITE THE PUBLISHED VERSION

http://dx.doi.org/10.1209/epl/i2002-00137-2

PUBLISHER

IOP Press / @ EDP Sciences

VERSION

AM (Accepted Manuscript)

\section{PUBLISHER STATEMENT}

This work is made available according to the conditions of the Creative Commons Attribution-NonCommercialNoDerivatives 4.0 International (CC BY-NC-ND 4.0) licence. Full details of this licence are available at: https://creativecommons.org/licenses/by-nc-nd/4.0/

\section{LICENCE}

CC BY-NC-ND 4.0

\section{REPOSITORY RECORD}

Archer, Andrew J., Robert Evans, and Roland Roth. 2019. "Microscopic Theory of Solvent-mediated Longrange Forces: Influence of Wetting”. figshare. https://hdl.handle.net/2134/16004. 
Europhysics Letters

PREPRINT

\title{
Microscopic theory of solvent mediated long range forces: influence of wetting
}

\author{
A.J. Archer ${ }^{1}$, R. Evans ${ }^{1}$ and R. Roth ${ }^{2,3}$ \\ 1 H.H. Wills Physics Laboratory, University of Bristol - Bristol BS8 1TL, UK \\ 2 Max-Plank Institut für Metallforschung - Heisenbergstr. 1, 70569 Stuttgart, Germany \\ 3 ITAP, Universität Stuttgart - Pfaffenwaldring 57, 70569 Stuttgart, Germany
}

PACS. 61.20Gy - Theory and models of liquid structure.

PACS. 82.70Dd - Colloids.

\begin{abstract}
We show that a general density functional approach for calculating the force between two big particles immersed in a solvent of smaller ones can describe systems that exhibit fluid-fluid phase separation: the theory captures effects of strong adsorption (wetting) and of critical fluctuations in the solvent. We illustrate the approach for the Gaussian core model, a simple model of a polymer mixture in solution and find extremely attractive, long ranged solvent mediated potentials between the big particles for state points lying close to the binodal, on the side where the solvent is poor in the species which is favoured by the big particles.
\end{abstract}

Determining the effective force between two (big) particles immersed in a solvent constitutes a canonical problem in condensed matter science. From the statistical mechanics viewpoint one should integrate out the relevant degrees of freedom of the solvent particles in order to obtain the the effective pair potential [1]. In the case of colloidal systems, where the colloidal component is very much larger than the particles constituting the solvent, the description of the complex, multi-component system in terms of an effective colloid-colloid potential forms a cornerstone of the subject. Well-known examples are the DLVO potential for charge stabilized systems and the hard-sphere potential for sterically stabilized systems [2]. The effective potential between colloids exhibits new features if particles of intermediate size, such as non-adsorbing polymer or small colloids, are added to the solvent. When two big colloids $(b)$ are sufficiently close that the intermediate sized particles are depleted from the region between the big colloids the effective $b b$ potential, $V_{b b}^{\text {eff }}(r)$, can exhibit entropically driven attraction. Within the Asakura-Oosawa (AO) model [3], where the colloids are hard spheres and the polymer is treated as ideal (inter-penetrating and non-interacting) the resulting depletion potential is purely attractive and its finite range is equal to the 'diameter' of the polymer coil. For mixtures of hard-sphere like colloids the effective potential between the bigger colloids exhibits both attraction and repulsion; short ranged correlations arising from the packing of the smaller colloids gives rise to an exponentially damped, oscillatory effective potential whose decay length is simply the bulk correlation length [1], in keeping with the

(c) EDP Sciences 
fact that $V_{b b}^{e f f}(r)=-k_{B} T \ln g_{b b}(r)$, where $T$ is the temperature and $g_{b b}(r)$ is the $b b$ radial distribution function of the mixture infinitely dilute in species $b$.

In this letter we investigate more generally the influence of correlations in the solvent on the nature of effective forces between two big particles. We suppose the solvent has a phase diagram such as that shown in fig. 1. The main features are: (i) possible phase separation into coexisting liquid and vapour phases, if the solvent is a pure system, or into two coexisting fluid phases, if the solvent is a binary mixture; (ii) a wetting point (denoted $W$ in fig. 1) below which macroscopically thick wetting layers of the coexisting phase can grow on a planar wall upon approaching coexistence along a path such as that denoted by $A$ [5, 6 ; ; (iii) a critical point $C$ at which the correlations in the solvent become macroscopically long ranged, i.e. the correlation length diverges.

For states well-removed from the coexistence curve (binodal) correlations in the solvent are short ranged, and the solvent mediated (SM) forces should also be short ranged. Qualitatively different features should arise in $V_{b b}^{e f f}(r)$ when long-ranged correlations occur. There are two obvious mechanisms for such behaviour. First, critical fluctuations of the solvent should give rise to long-ranged forces between plates or big particles [7]. These 'critical Casimir forces' are expected to induce flocculation of colloids suspended in near-critical solvents. The second mechanism, and the one of primary interest here, is that associated with the growth of wetting films around plates or sufficiently large particles. We consider a path such as $A$ in fig. 1, which lies below the wetting point $W$. On approaching the binodal the coexisting phase (small concentration $x$ ) will wet completely the interface between a planar wall and the bulk fluid phase (large $x$ ). In the case of large particles, made of the same material as the wall, thick adsorbed films will develop but, because of the finite radius of curvature, films will remain of finite thickness even at coexistence [5]. One might expect to find long ranged SM forces arising from the presence of such 'wetting' films. Indeed wetting induced effective potentials between spherical particles immersed in a one-component fluid have been calculated using an interface displacement (sharp-kink) description of the solvent density distribution [8]. Although wellsuited to very big particles and thermodynamic states very close to coexistence, where very thick films can develop, and to systems where dispersion forces dominate, the approach of ref. [8] is less well-suited to situations where the particles are not enormous, so that the films are thinner, and where dispersion forces do not dominate. Our present approach, whilst also based on density functional theory (DFT), implements the general method for including correlation effects developed in the calculation of depletion potentials [4. Since it makes no particular assumption about the form of the density distributions it is applicable for all (fluid) states, including those close to the critical point where a sharp-kink approximation is inappropriate. We first treat one big particle as an external potential by fixing its position at the origin and then calculate the equilibrium density profile(s) of the solvent particles in this external potential. Thus we input a fully microscopic description of the "wetting film' (or of the long range decay of the density profile(s) if we are near the critical point). The next step is to calculate the SM potential by inserting a second big particle using the potential distribution theorem [4,9]. To this end we require a functional capable of describing a mixture of the solvent and big particles that is reliable in the limit of vanishing density of big particles [4]. There has been progress in this regard. The Rosenfeld functional [10] for hard sphere mixtures was used successfully to calculate depletion potentials for a wide range of size ratios [4]. However, hard-sphere mixtures do not exhibit fluid-fluid demixing. Recently a DFT for the AO model of a colloid-polymer mixture was derived [1]. For certain size ratios the mixture undergoes phase separation into colloid rich and colloid poor fluid phases and the DFT predicts entropically driven complete wetting of a planar hard wall by the colloid 


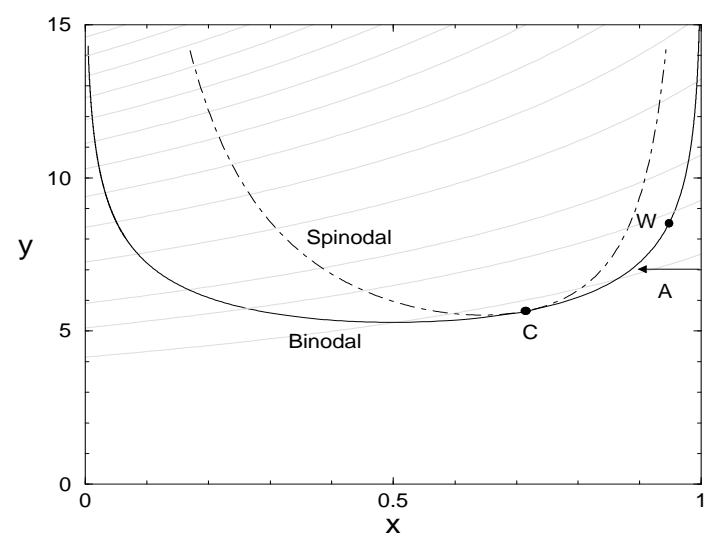

Fig. 1 - Phase diagram of a solvent exhibiting fluid-fluid phase separation. For gas-liquid phase separation $x$ corresponds to the fluid density and $y$ to inverse temperature. In the case of a binary mixture, $x$ is the relative concentration of one of the species, and $y$ corresponds to the temperature or, alternatively, to the total bulk density, $\rho^{0}$. For the present binary fluid of GCM particles $x=\rho_{2}^{0} / \rho^{0}$, where $\rho_{2}^{0}$ is the density of species 2 , and $y=\rho^{0} R_{11}^{3}$ where $R_{11}$ is the radius of the larger species 1 . The pair potential parameters are: $\epsilon_{12} / \epsilon_{11}=0.944$ and $R_{22} / R_{11}=0.665$ which is equivalent to a mixture of two polymers with length ratio $2: 1$. The gray lines are isobars, the lowest is at reduced pressure $P \beta R_{11}^{3}=100$, the next at $P \beta R_{11}^{3}=150$, then 200 with the subsequent ones increasing in increments of 100. $W$ denotes the wetting point, i.e. the surface phase transition, below which a thick wetting film of the phase rich in species 1 can grow on a wall or on a big particle immersed in the binary mixture at a state near the right hand binodal. Arrow $A$ indicates the path along which the density profiles in Fig. 2 are calculated. $C$ denotes the critical point.

rich phase [12]. It is straightforward to generalize the functional to include an additional component of big hard spheres at which 'wetting' films develop as coexistence is approached from the colloid poor side of the binodal. Results for effective potentials between the big hard spheres will be presented elsewhere [13].

Another, extremely simple functional [14, 15] for a mixture of soft core repulsive Gaussian particles, which models the interaction between the centers of mass of polymers in solution [2], was also found to be capable of treating mixtures with large size asymmetries. The important feature for the present study is that whilst the one component Gaussian core model (GCM) does not phase separate, a binary mixture of two different sized GCM particles does separate into two fluid phases [14, 15] and displays wetting transitions [6] for certain, purely repulsive, planar walls. The phase diagram of fig. 1 refers to the particular binary GCM mixture whose wetting properties were studied in ref. [6]. Here we consider a ternary GCM mixture with the density of the biggest (repulsive) Gaussian particles $\rho_{b}^{0} \rightarrow 0$.

We outline the general method used to calculate the SM potential - a more detailed account can be found in ref. 淊. The SM potential between two objects $a$ and $b$ is defined by $W_{a b}\left(\mathbf{r}_{b}\right)=\Omega\left(\mathbf{r}_{b}\right)-\Omega\left(\mathbf{r}_{b} \rightarrow \infty\right)$, where $\Omega\left(\mathbf{r}_{b}\right)$ is the grand potential of the solvent with object $a$ fixed at $\mathbf{r}_{a}=0$ and object $b$ at $\mathbf{r}_{b}$. Using the potential distribution theorem this difference in grand potential can be written in terms of the one-body direct correlation function $c_{b}^{(1)}$ in the limit of the chemical potential of species $b, \mu_{b} \rightarrow-\infty$, equivalent to the limit of the bulk density, $\rho_{b}^{0} \rightarrow 0$ [画:

$$
\beta W_{a b}\left(\mathbf{r}_{b}\right)=c_{b}^{(1)}\left(\mathbf{r}_{b} \rightarrow \infty ; \mu_{b} \rightarrow-\infty\right)-c_{b}^{(1)}\left(\mathbf{r}_{b} ; \mu_{b} \rightarrow-\infty\right),
$$


where $\beta=1 / k_{B} T$. The one-body correlation function $c_{b}^{(1)}(\mathbf{r})$ is given within DFT 16] by $c_{b}^{(1)}(\mathbf{r})=-\beta \delta \mathcal{F}_{e x}\left[\left\{\rho_{i}\right\}\right] / \delta \rho_{b}(\mathbf{r})$, where $\mathcal{F}_{\text {ex }}$ is the excess (over ideal gas) Helmholtz free energy functional of the mixture of the solvent and the big particles, $\rho_{i}$ denotes the density profile of species $i$, with $\rho_{b}$ that of the big particles. The effective potential between two big particles is then the sum of the bare interaction $v_{b b}(r)$ and the SM potential: $V_{b b}^{e f f}(r)=v_{b b}(r)+W_{b b}(r)$. In order to implement eq. (1), an appropriate approximation for $\mathcal{F}_{e x}$ for the mixture under consideration must be specified.

We focus on the GCM and refer the reader interested in its origins to ref. [2]. The structure of the bulk GCM fluid at high densities is very different from that of hard core fluids, since the particle cores are soft and hence can overlap. For a mixture the pair potential between particles is given by $\beta v_{i j}(r)=\epsilon_{i j}^{*} \exp \left(-r^{2} / R_{i j}^{2}\right)$, where for polymers in an athermal solvent at room temperature $\epsilon_{i j}^{*} \simeq 2$ and $R_{i i}$ is roughly the radius of gyration. The radius of cross interaction is usually taken to be $R_{i j}^{2}=\left(R_{i i}^{2}+R_{j j}^{2}\right) / 2$ [14, 17]. The cross interaction energy parameter $\epsilon_{i \neq j}^{*}<\epsilon_{i i}^{*}$ reflects the way a small polymer coil can overlap with a larger polymer with a smaller energy penalty than for a polymer of the same size. The particular values we choose are $\epsilon_{11}^{*}=\epsilon_{22}^{*}=2, \epsilon_{12}^{*}=1.89, R_{22} / R_{11}=0.665$ [6, 14, 15] and $\epsilon_{b 1}^{*}=1.0, \epsilon_{b 2}^{*}=0.8$ and $R_{b b} / R_{11}=7.0$, resulting in radii of cross interaction of $R_{b 1} / R_{11}=5.0$ and $R_{b 2} / R_{11}=4.97$. At densities where the soft cores of the GCM particles start to overlap, such that a particle interacts with a large number of its neighbours, the fluid behaves as a mean-field fluid [2]. The simple random phase approximation (RPA), which states that the pair direct correlation function is simply $c_{i j}^{(2)}(r)=-\beta v_{i j}(r)$, accounts well for the bulk correlations at these high densities [2, 14, 15]. The excess free energy functional which generates the RPA for an arbitrary number of components is

$$
\mathcal{F}_{e x}\left[\left\{\rho_{i}\right\}\right]=\frac{1}{2} \sum_{i j} \int \mathrm{d} \mathbf{r}_{1} \int \mathrm{d} \mathbf{r}_{2} \rho_{i}\left(\mathbf{r}_{1}\right) \rho_{j}\left(\mathbf{r}_{2}\right) v_{i j}\left(\left|\mathbf{r}_{1}-\mathbf{r}_{2}\right|\right) .
$$

since $c_{i j}^{(2)}\left(\mathbf{r}_{1}, \mathbf{r}_{2}\right)=-\beta \delta^{2} \mathcal{F}_{e x} / \delta \rho_{i}\left(\mathbf{r}_{1}\right) \delta \rho_{j}\left(\mathbf{r}_{2}\right)$ [16]. Using this (RPA) functional for the ternary mixture, with $i, j=1,2$ and $b$, in eq. (1) the SM potential reduces to

$$
W_{b b}\left(\mathbf{r}_{1}\right)=-\sum_{i=1}^{2} \int \mathrm{d} \mathbf{r}_{2}\left(\rho_{i}\left(\mathbf{r}_{2}\right)-\rho_{i}^{0}\right) v_{b i}\left(\left|\mathbf{r}_{1}-\mathbf{r}_{2}\right|\right),
$$

where the sum now runs over the small particle species only since $\rho_{b}^{0} \rightarrow 0$. The profiles $\rho_{i}(\mathbf{r})$, $i=1,2$, are the density profiles of the small particle species obtained by solving the EulerLagrange equations for the situation where the big particle exerts an external potential on the binary mixture 顿. $\rho_{i}^{0}$ are the small particle bulk densities. Near the binodal (for example on path $A$, fig. 1), the density profiles of the smaller particle species will reflect the formation of a thick adsorbed film around a single big particle, and so within the RPA treatment we have a prescription for including at least some of the effects of wetting on the SM potential.

We study the same binary mixture of GCM particles, equivalent to a binary mixture of polymers of length ratio $2: 1$, as in refs. [6, 15]. Into this phase separating binary mixture we now add the third, much bigger species of GCM particle with $R_{b b} / R_{11}=7.0$. In fig. 2 we show the density profiles of both components of the binary solvent calculated along path $A$ in fig. 1, where the total bulk density is constant, $\rho^{0} R_{11}^{3}=7.0$. As the the binodal is approached the shape of the profiles changes dramatically as the thick adsorbed film develops. For concentrations $x$ close to the coexistence value, $x_{c o e x}$, the profiles have a flat central portion where the densities take values roughly similar to those in the coexisting bulk phase 
rich in species 1 . We denote these $\rho_{i}^{\text {coex }}, i=1,2$. In this regime the adsorbed film extends beyond the radius $R_{b b}$ of the big particle fixed at the origin but because of curvature it remains of finite extent at $x=x_{\text {coex }}$. In the opposite regime, near $x=1$, the profiles are Gaussianlike. Indeed in the limit of a pure solvent of GCM particles of species 2 the profile can be approximated extremely accurately by the simple ansatz $\rho_{2}(r)=\rho_{2}^{0}-\rho^{*} \exp \left(-r^{2} / R_{b 2}^{2}\right)$ with $\rho^{*}=\rho_{2}^{0} \epsilon_{b 2}^{*} /\left(1+\rho_{2}^{0} \pi^{3 / 2} \epsilon_{22}^{*} R_{22}^{3}\right)$, provided $R_{b b} / R_{22} \gg 1$ [18]. When this ansatz is inserted into eq. (3) we find that

$$
\beta W_{b b}^{\text {pure }}(r)=-(\pi / 2)^{3 / 2} \epsilon_{b 2}^{*} \rho^{*} R_{b 2}^{3} \exp \left(-r^{2} / 2 R_{b 2}^{2}\right)
$$

which lies on top of the full numerical result (top curve in fig. 3). Thus even when there is no adsorbed film the SM potential is strongly attractive $\left(W_{b b}(r=0) \simeq-45 k_{B} T\right.$ for $\left.\rho_{2}^{0} R_{11}^{3}=7.0\right)$. The large amplitude arises from the factor $\rho^{*} R_{b 2}^{3}$ in eq. (4), which is roughly the number of small particles expelled from the volume of the big one. This factor is large in these high density states. As we move along path $A$ (fig. 1), adding more of species 1 to the host fluid, we find that as the binodal is approached the SM potential, obtained by inserting the profiles of fig. 2 into eq. (3), becomes longer ranged and deeper (see fig. 3). For example, when $x=\rho_{2}^{0} / \rho^{0}=0.887$ then $W_{b b}\left(r=2 R_{b b}\right) \simeq-30 k_{B} T$ and $W_{b b}(r=0) \simeq-650 k_{B} T$. Note that the underlying (bare) repulsive big-big potential $v_{b b}(r)$ will be negligible in comparison with such strongly attractive SM potentials. It is clear that enormous attractive interactions are generated in this mixture where all the bare interparticle potentials are purely repulsive.

Why does the presence of thick adsorbed films induce such deep SM potentials? In order to obtain some insight we can approximate the density profiles of the solvent using the so-called sharp-kink (sk) approximation [5], i.e. $\rho_{i}(r)=\rho_{i}^{\text {coex }}$ for $0<r \leq l$ and $\rho_{i}(r)=\rho_{i}^{0}$ for $r>l$, where $l$ is the thickness of the adsorbed film. On substituting this approximation for $\rho_{i}(r)$ into eq. (3) we find that

$$
\beta W_{b b}^{s k}(r)=\frac{\pi}{2} \sum_{i=1}^{2} \Delta \rho_{i} \epsilon_{b i}^{*} R_{b i}^{3}\left\{\sqrt{\pi}\left[\operatorname{erf}\left(r_{-}\right)+\operatorname{erf}\left(r_{+}\right)\right]-\frac{R_{b i}}{r}\left(e^{-r_{-}^{2}}-e^{-r_{+}^{2}}\right)\right\}
$$

where $\Delta \rho_{i}=\left(\rho_{i}^{\text {coex }}-\rho_{i}^{0}\right)$ is the difference in coexisting density for species $i, \operatorname{erf}(x)=$ $(2 / \sqrt{\pi}) \int_{0}^{x} d t \exp \left(-t^{2}\right)$ is the error function and $r_{ \pm}=(l \pm r) / R_{b i}$. The energy scale at coexistence is set by $\beta \Delta \Omega \equiv c_{b}^{(1)}\left(\infty ;\left\{\rho_{i}^{\text {coex }}\right\}\right)-c_{b}^{(1)}\left(\infty ;\left\{\rho_{i}^{0}\right\}\right)=-\sum_{i} \Delta \rho_{i} \pi^{3 / 2} \epsilon_{b i}^{*} R_{b i}^{3}>0$ [18], the difference in the grand potential between inserting a single large GCM particle in the bulk fluid near the phase boundary (bulk densities $\rho_{i}^{0}$ ) and inserting the large particle in the bulk coexisting phase (the phase adsorbing on the big particle, densities $\rho_{i}^{\text {coex }}$ ). For a thick enough adsorbed film around the big particle, there will be a region inside the film where we can insert the second big particle sufficiently far away from both the fluid-fluid interface and from the central big particle at the origin, that the grand potential for inserting the big particle is approximately that of inserting it into the bulk of the coexisting phase. For the point on the binodal at $\rho^{0} R_{11}^{3}=7.0$ (the intersection with path $A$ fig. 11) we find $\beta \Delta \Omega=627$, the large value arising mainly from the high values of $\left(R_{b i} / R_{11}\right)^{3}$, which should be a general feature of the GCM. The comparison between the sharp kink approximation and the full calculation is shown in the inset to fig. 3, taking a film thickness $l / R_{11}=9.5$. We find that eq. (5) captures the gross features of the SM potential calculated numerically for $x$ very close to $x_{\text {coex }}$. In other types of fluids the quantity $\beta \Delta \Omega$ should also set the energy scale at coexistence but its form will depend on the particular fluid. For example in the AO model the depth of the depletion potential scales roughly as $R_{c c} / R_{p p}$, where $c$ refers to the hard-sphere colloid and $p$ to ideal polymer. We expect crossover to $\left(R_{b b} / R_{p p}\right)^{2}$ scaling when two very big hard-spheres are immersed in the colloid poor phase and are 'wet' by the colloid rich phase at coexistence [13]. 


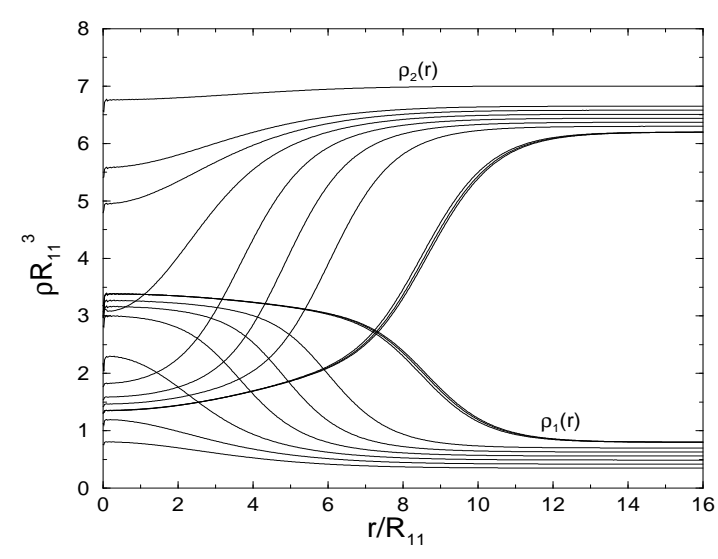

Fig. 2

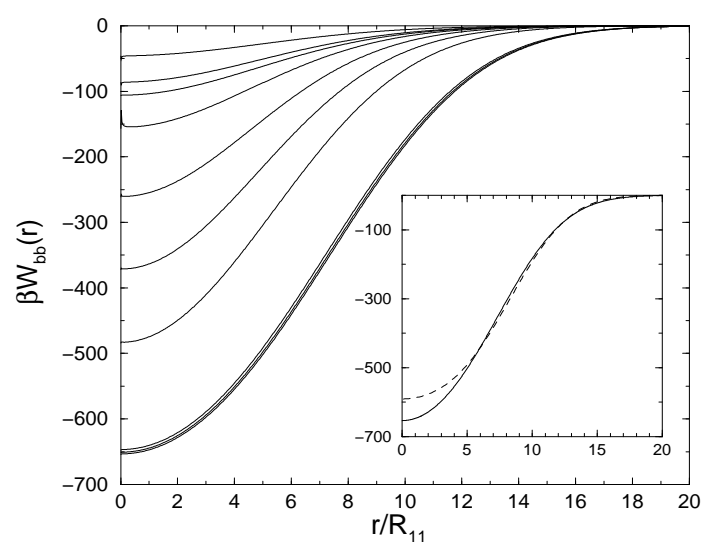

Fig. 3

Fig. 2 - The density profiles of a binary solvent of small GCM particles, of radii $R_{11}$ and $R_{22}$ with $R_{22} / R_{11}=0.665$, around a large GCM test particle with $R_{b b} / R_{11}=7.0$, calculated at constant total bulk density $\rho^{0} R_{11}^{3}=7.0$ i.e. along path $A$ in fig. 田, at concentration $x=\rho_{2}^{0} / \rho^{0}=1.0,0.95,0.94$, $0.93,0.92,0.91,0.9,0.886,0.8857$ and $0.8855\left(x_{\text {coex }} .=0.8854\right)$. The top set of profiles are those of the smaller of the two species, species 2 . The very top one is for $x=1.0$ and the one below is for 0.95 etc. The set of profiles at the bottom correspond to species 1 ; the very lowest profile is at $x=0.95$ (for $x=1.0$ the profile is $\rho_{1}(r)=0$ ), the one above is for 0.94 etc. Note the pronounced change in shape of the density profiles from roughly Gaussian away from coexistence, to a non-Gaussian shape, with a flat portion near the origin and the free interface-like 'knee', close to coexistence. The latter shape signals a thick adsorbed film of the coexisting phase, rich in species 1 , growing around the big GCM particle.

Fig. 3 - The SM potential between two big GCM particles with radius $R_{b b} / R_{11}=7.0$ as calculated from the density profiles in Fig. The potentials correspond to concentration $x=1.0,0.95,0.94$, $0.93,0.92,0.91,0.9,0.886,0.8857$ and 0.8855 , going from top to bottom $\left(x_{\text {coex }}=0.8854\right)$. The SM potential increases in depth and range as the binodal is approached. The energy scale close to coexistence is set by the difference in the grand potential between inserting a single big particle in the bulk and inserting it into the bulk coexisting phase which constitutes the adsorbed film and for the GCM (see text) this results in particularly deep potentials. In the inset we re-plot the SM potential calculated at $x=0.8855$ (solid line) along with our analytic approximation, eq. (5) (dashed line), with $l / R_{11}=9.5$.

We have also calculated the SM potential for states in the vicinity of the critical point $C$ in fig. 1. When the bulk correlation length of the binary GCM is several times $R_{11}, W_{b b}(r)$ decays slowly with separation $r$, i.e. the SM force becomes very long ranged. Results will be reported elsewhere [18]. Note that our general procedure is formally equivalent to calculating the effective $b b$ potential from the logarithm of $g_{b b}(r)$, in the limit $\rho_{b}^{0} \rightarrow 0$, via the test particle route [4]. Since the procedure can also be used to calculate the SM potential between a test particle and a planar wall (or another fixed object) [4] we can investigate the influence of wetting films on effective wall-particle forces for arbitrary sizes of test particle.

Some impetus for theories [8] of wetting-induced interaction potentials comes from experimental observations of striking flocculation phenomena for colloidal particles immersed in a binary liquid mixture close to the binodal [19]. Flocculation appears to take place on the side of the phase diagram where the mixture is poor in the species which is favoured by the colloid. This is where we predict strongly attractive forces. Although the precise interpretation of the 
experiments remains somewhat controversial - critical Casimir forces and other mechanisms specific to the experimental systems might also play a role - it is clear that wetting can have a profound effect on effective colloid-colloid interactions.

The approach we have outlined here is a step towards a general microscopic theory of solvent mediated interactions which can incorporate the effects of both wetting and bulk criticality, albeit at a mean-field level, for a wide range of particle sizes. As such it complements the mesoscopic approach of ref. [8] and some other approaches used in studies of near-critical solvents [20]. We find that for a particular model, namely a mixture of repulsive Gaussian core particles, the SM interactions between the big particles can be long ranged and very strongly attractive near the binodal. We expect to find similar long-ranged attractive forces induced by wetting in all solvents exhibiting fluid-fluid phase separation.

We benefitted from helpful discussions with J.M. Brader, M. Schmidt, and H. Löwen. AJA was supported by an EPSRC studentship.

\section{REFERENCES}

[1] W.G McMillan and J.E. Mayer, J. Chem. Phys., 13 (1945) 276.

[2] For a recent review of effective interactions in colloidal and other soft matter systems see C.N. Likos, Phys. Reports, 348 (2001) 267. For charged colloids see J-.P. HANsEn and H. LöwEn, Ann. Rev. Phys. Chem., 51 (2000) 209.

[3] S. Asakura and F. Oosawa, J. Chem. Phys., 22 (1954) 1255.

[4] R. Roth, R. Evans, and S. Dietrich, Phys. Rev. E, 62 (2000) 5360 and references therein.

[5] see e.g. S. Dietrich, Phase Transitions and Critical Phenomena, edited by C. Domb and J.L. Lebowitz, Vol. 12 (London: Academic) 1988, p. 1.

[6] A.J. Archer and R. Evans, J. Phys.: Condens. Matter, 14 (2002) 1131.

[7] M.E. Fisher and P.G. De Gennes, C.R. Acad. Sc. Paris B, 287 (1978) 207.

[8] C. Bauer, T. Bieker, and S. Dietrich, Phys. Rev. E, 62 (2000) 5324.

[9] J.R. Henderson, Mol. Phys., 50 (1983) 741.

[10] Y. Rosenfeld, Phys. Rev. Lett., 63 (1989) 980.

[11] M. Schmidt, H. Löwen, J.M. Brader and R. Evans, Phys. Rev. Lett., 85 (2000) 1934.

[12] J.M. Brader, R. Evans, M. Schmidt and H. Löwen, J. Phys. Cond. Matt., 14 (2002) L1.

[13] R. Roth, and R. Evans, in preparation.

[14] A.A. Louis, P.G. Bolhuis, and J-P. Hansen, Phys. Rev. E, 62 (2000) 7961.

[15] A.J. Archer and R. Evans, Phys. Rev. E, 64 (2001) 041501.

[16] R. Evans, Adv. Phys, 28 (1979) 143.

[17] J. Dautenhahn and C.K. Hall, Macromolecules, 27 (1994) 5399.

[18] A.J. Archer and R. Evans, in preparation.

[19] Many of these experiments were carried out by D. Beysens and co-workers. For a comprehensive list of references and a summary of attempts to explain the data see T. BIEKER, and S. DiETRICH, Physica A, 252 (1998) 85 and ref. [8].

[20] F. Schlesener, A. Hanke and S. Dietrich, cond-mat/0202532, and references therein. 